\title{
Unusually Rapid Development of Pulmonary Hypertension and Right Ventricular Failure after COVID-19 Pneumonia
}

\author{
Christel MP van Dongen ${ }^{1}$, Marlou THF Janssen², Robrecht PJ van der Horst ${ }^{2,3}$, Dave JW van Kraaij ${ }^{4}$, \\ Ralph HRM Peeters ${ }^{1}$, Leon M van den Toorn ${ }^{5}$, Rémy LM Mostard ${ }^{2}$ \\ ${ }^{1}$ Department of Rheumatology, Zuyderland Medical Center, Heerlen-Sittard, the Netherlands \\ ${ }^{2}$ Department of Pulmonary Medicine, Zuyderland Medical Center, Heerlen-Sittard, the Netherlands \\ ${ }^{3}$ Department of Intensive Care Medicine, Zuyderland Medical Center, Heerlen-Sittard, the Netherlands \\ ${ }^{4}$ Department of Cardiology, Zuyderland Medical Center, Heerlen-Sittard, the Netherlands \\ ${ }^{5}$ Department of Pulmonary Medicine, Erasmus Medical Center, Rotterdam, the Netherlands
}

Doi: 10.12890/2020_001784 - European Journal of Case Reports in Internal Medicine - ㅇ EFIM 2020

Received: 08/06/2020

Accepted: $13 / 06 / 2020$

Published: $17 / 06 / 2020$

How to cite this article: van Dongen CMP, Janssen MTHF, van der Horst RPJ, van Kraaij DJW, Peeters RHRM. van den Toorn LM, Mostard RLM. Unusually rapid development of pulmonary hypertension and right ventricular failure after COVID-19 pneumonia. EJCRIM 2020;7: doi:10.12890/2020_001784.

Conflicts of Interests: The Authors declare that there are no competing interests.

This article is licensed under a Commons Attribution Non-Commercial 4.0 License

\section{ABSTRACT}

COVID-19 is a novel viral disease caused by SARS-CoV-2. The mid- and long-term outcomes have not yet been determined. COVID-19 infection is increasingly being associated with systemic and multi-organ involvement, encompassing cytokine release syndrome and thromboembolic, vascular and cardiac events. The patient described experienced unusually rapid development of pulmonary hypertension $(\mathrm{PH})$ and right ventricular failure after recent severe COVID-19 pneumonia with cytokine release syndrome, which initially was successfully treated with methylprednisolone and tocilizumab. The development of pulmonary hypertension and right ventricular failure - in the absence of emboli on multiple CT angiograms - was most likely caused by progressive pulmonary parenchymal abnormalities combined with microvascular damage of the pulmonary arteries (group III and IV pulmonary hypertension, respectively). To the best of our knowledge, these complications have not previously been described and therefore awareness of PH as a complication of COVID-19 is warranted.

\section{LEARNING POINTS}

- COVID-19 increasingly presents with systemic and multi-organ involvement with vascular, thromboembolic and cardiac events.

- Patients with severe COVID-19 pneumonia and concomitant cytokine release syndrome may be particularly at risk for the development of secondary pulmonary hypertension and right ventricular failure.

- Pulmonary hypertension can develop unusually rapidly following COVID-19 pneumonia and probably results from progressive pulmonary interstitial and microvascular abnormalities due to COVID-19.

\section{KEYWORDS}

COVID-19, coagulopathy, microvascular damage, pulmonary hypertension, right ventricular failure

\section{INTRODUCTION}

COVID-19 is a novel disease with a broad and expanding clinical presentation. There is growing evidence of cytokine release syndrome (CRS) in patients with severe disease ${ }^{[1]}$. Furthermore, hypercoagulability due to this pro-inflammatory state is very prevalent in these patients and leads to thromboembolic events ${ }^{[2]}$. COVID-19 infection complicated by CRS and hypercoagulability with potential multi-organ 
complications is increasingly recognised. With ongoing disease and rising numbers of COVID-19 patients being discharged from hospital, the focus will shift towards mid- and long-term follow-up and complications. This case report describes a patient with unusually rapid development of life-threatening pulmonary hypertension (PH) following earlier hospitalisation and successful treatment for severe CRS caused by COVID-19 infection.

\section{CASE DESCRIPTION}

A 60-year-old man, with a medical history of minor myocardial infarction with no abnormalities on coronary angiography 7 years earlier, was admitted to our intensive care unit (ICU) for severe COVID-19 pneumonia in April 2020. He was treated with mechanical ventilation, ceftriaxone, chloroquine and low-molecular-weight heparin in prophylactic doses, since CT angiography (CTA) revealed no signs of pulmonary embolism (PE). Because of associated CRS, treatment with methylprednisolone was initiated, followed by tocilizumab. The patient was weaned off ventilation after a few days. Within 2 weeks he was discharged to a rehabilitation centre, with $1 \mathrm{l} / \mathrm{min}$ nasal oxygen support.

However, 10 days after discharge, he was re-admitted to the ICU because of progressive dyspnoea and severe hypoxaemia, requiring highflow nasal oxygen. Laboratory tests revealed no recurrence of CRS (C-reactive protein $32 \mathrm{mg} / \mathrm{l}$, ferritin $413 \mu \mathrm{g} / \mathrm{l}$ and D-dimer $659 \mu \mathrm{g} / \mathrm{I}$ ) or ongoing viral pneumonia (SARS-CoV-2 PCR was twice negative on Eswab and bronchoalveolar lavage fluid). Repeated CTA also proved negative for PE, but demonstrated progressive interstitial pulmonary abnormalities. Signs of cardiac decompensation or acute coronary syndrome were absent (including low NT-proBNP and troponin-T). During the first days after re-admission, oxygenation worsened, with diuretics not having any effect. Eventually the patient was intubated, requiring high doses of sedation, fluids and vasopressors. The negative response to diuretics and slightly enlarged P-waves on electrocardiography required further cardiac evaluation. Cardiac ultrasound confirmed normal left ventricular function without valvular disease, but revealed a grossly enlarged right ventricle (RV) with a flattened interventricular septum ('Delta sign'). Based on tricuspid regurgitation velocity and inferior caval vein diameter and collapse, RV systolic pressure (RVSP) was estimated to be $60-65 \mathrm{mmHg}$ (Figs. 1 and 2), indicating $\mathrm{PH}$.

Cardiogenic shock was suspected because of an acute rise in serum creatinine and serum liver transaminase levels. Because of the patient's critical condition, treatment with sildenafil and methylprednisolone was initiated as a pragmatic approach. Repeated echocardiography 10 days later showed disappearance of the Delta sign, and diminished RV and inferior caval vein dimensions with a RVSP estimated at 30-35 $\mathrm{mmHg}$. Currently, the patient is still on mechanical ventilation.

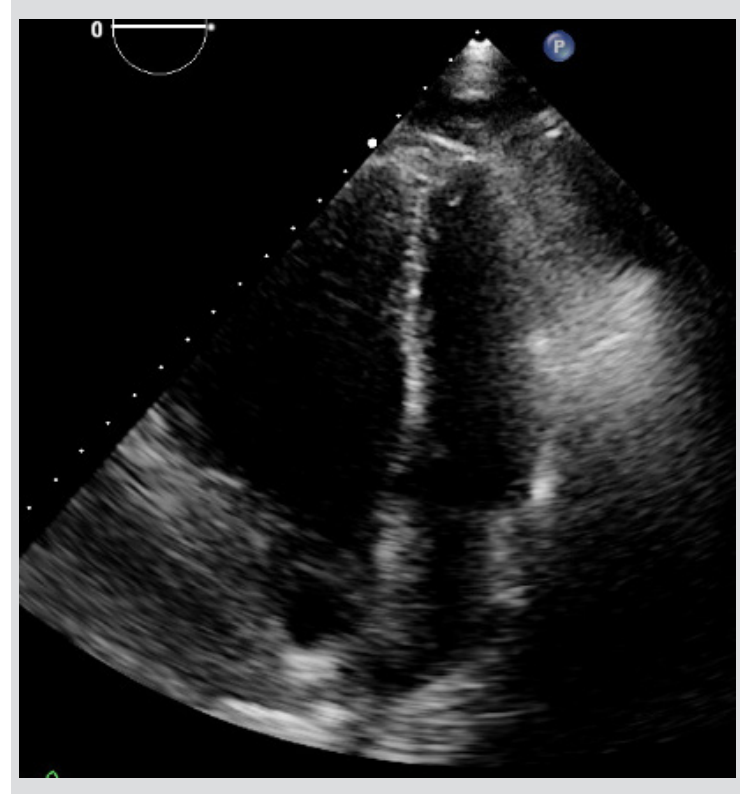

Figure 1. Apical end-systolic four-chamber view of the heart, showing a grossly enlarged right ventricle with leftward displacement of the interventricular septum

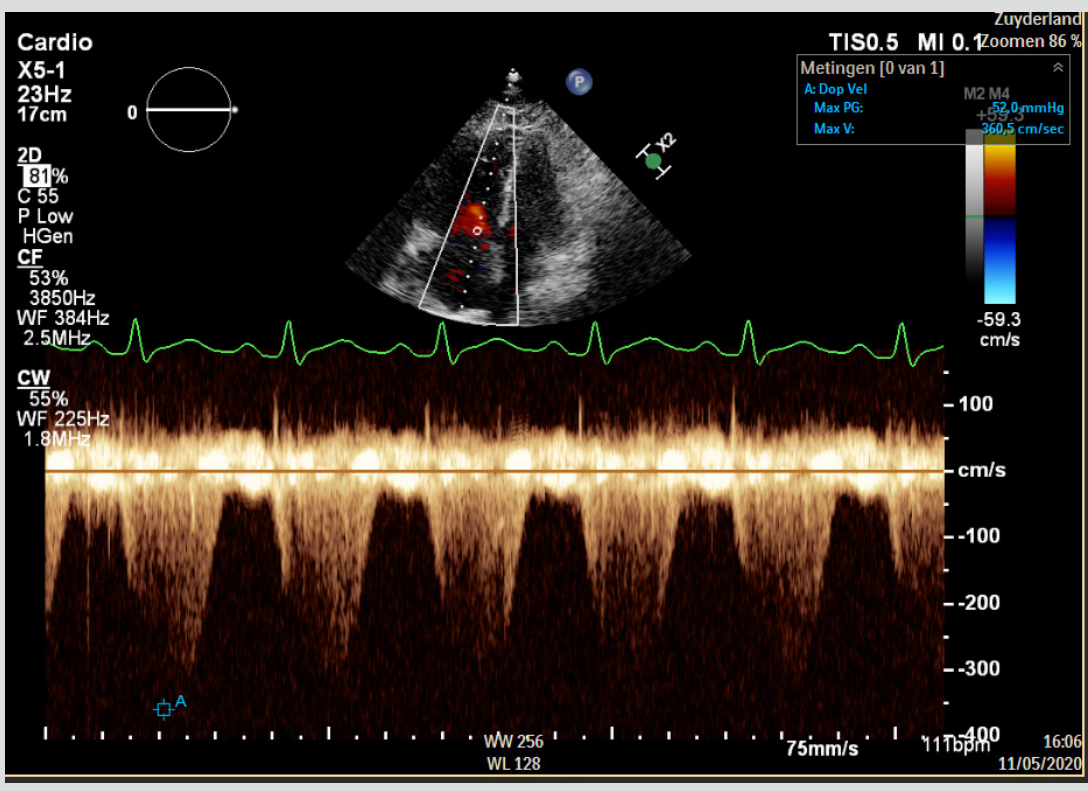

Figure 2. Systolic continuous-wave Doppler signal showing tricuspid regurgitation. As a dilated inferior caval vein also showed insufficient collapse, right ventricular systolic pressures were estimated to be $60-65 \mathrm{mmHg}$ 


\section{DISCUSSION}

COVID-19 pneumonia often causes extensive bilateral lung damage, eventually leading to pulmonary fibrosis in some patients ${ }^{[3]}$. COVID-19 appears to provoke a hypercoagulable state and $\mathrm{PE}{ }^{[4]}$. Coagulopathy is most likely explained by immunothrombotic pathways and by complement-mediated microvascular injury, reported in autopsies ${ }^{[5,6]}$. Diffuse alveolar and interstitial damage, inflammation and extensive pulmonary macrophage activation, compatible with CRS, all contribute to pulmonary intravascular coagulopathy ${ }^{[7]}$. Dysregulation of the pulmonary innate immune response, like ACE2 receptor expression downregulation, as well as altered adaptive immune responses, are potential pathophysiological mechanisms for the development of PE in COVID-19 [7].

$\mathrm{PH}$ is usually classified into five groups ${ }^{[8]}$. In this patient, extensive autoimmune laboratory evaluation proved negative and no high-risk medication for the development of PH had been used previously, suggesting group I PH (idiopathic and heritable), so it was most unlikely that drug-induced, connective tissue disease was the cause of the PH. Furthermore, indications for group II (due to left heart disease) and group V PH (due to unclear or multifactorial mechanisms) were absent. Given the rapid onset of symptoms in this patient - after initial recovery from severe COVID-19 pneumonia with CRS - a combination of group III (due to obstructive or restrictive lung disease) and IV PH (due to pulmonary artery obstruction) is proposed. However, macro-vascular PE could not be confirmed with repeated CTA. Microvascular injury may have had an important role in the development of PH in the current case. In view of the association of COVID-19 with coagulopathy and microvascular disease, subsequent potential micro-PE as well as interstitial pulmonary damage, $\mathrm{PH}$ is likely to be a frequent complication of severe COVID-19 pneumonia and CRS. Furthermore, the patient described in this case report developed PH over a much shorter time period than usually expected considering the underlying pathophysiological mechanisms.

COVID-19 is a pulmonary viral infection which can result in severe systemic, multi-organ disease in some patients. The long-term consequences of this disease are not fully known, but are gradually emerging and being described. To the best of our knowledge, this is the first case report of a patient who developed PH unusually quickly after COVID-19 infection, probably due to residual marked pulmonary parenchymal abnormalities in combination with microvascular damage of the pulmonary arteries. Therefore, awareness of $\mathrm{PH}$ as a complication of COVID-19 is warranted.

\section{REFERENCES}

1. Mehta P, McAuley DF, Brown M, Sanchez E, Tattersall RS, Manson JJ. COVID-19: consider cytokine storm syndromes and immunosuppression. Lancet 2020;395(10229):10331034.

2. Levi M, Thachil J, Iba T, Levy JH. Coagulation abnormalities and thrombosis in patients with COVID-19. Lancet Haematol 2020;7(6):e438-e440.

3. Wu J, Pan J, Teng D, Xu X, Feng J, Chen YC. Interpretation of CT signs of 2019 novel coronavirus (COVID-19) pneumonia. Eur Radiol 2020 May 4;1-8. doi: 10.1007/s00330020-06915-5 [Epub ahead of print].

4. Klok FA, Kruip MJHA, van der Meer NJM, Arbous MS, Gommers DAMPJ, Kant KM, et al. Incidence of thrombotic complications in critically ill ICU patients with COVID-19. Thromb Res 2020;191:145-147.

5. Magro C, Mulvey JJ, Berlin D, Nuovo G, Salvatore S, Harp J, et al. Complement associated microvascular injury and thrombosis in the pathogenesis of severe COVID-19 infection: a report of five cases. Transl Res 2020;220:1-13.

6. Ackermann M, Verleden SE, Kuehnel M, Haverich A, Welte T, Laenger F, et al. Pulmonary vascular endothelialitis, thrombosis, and angiogenesis in Covid-19. N Engl J Med 2020 May 21. doi: 10.1056/NEJMoa2015432 [Epub ahead of print].

7. McGonagle D, O'Donnell JS, Sharif K, Emery P, Bridgewood C. Immune mechanisms of pulmonary intravascular coagulopathy in COVID-19 pneumonia. Lancet Rheumatol 2020 May 7. doi: 10.1016/S2665-9913(20)30121-1 [Epub ahead of print].

8. Simonneau G, Montani D, Celermajer DS, Denton CP, Gatzoulis MA, Krowka M, et al. Haemodynamic definitions and updated clinical classification of pulmonary hypertension. Eur Respir J 2019;53(1):1801913. 